\title{
Promoting and Assessing Student Metacognition in Physics
}

\author{
Alistair McInerny ${ }^{1}$, Andrew Boudreaux ${ }^{1}$, Mila Kryjevskaia ${ }^{2}$ and Sara Julin ${ }^{3}$ \\ ${ }^{1}$ Department of Physics and Astronomy, Western Washington University, 516 High St, Bellingham, WA 98225 \\ ${ }^{2}$ Department of Physics, North Dakota State University, 1211 Albrecht Blvd, Fargo, ND 58105 \\ ${ }^{3}$ Whatcom Community College, 237 W Kellogg Rd, Bellingham, WA, 98226
}

\begin{abstract}
A scaffolded metacognition activity was incorporated into the laboratory component of the introductory physics course at Western Washington University (WWU) and Whatcom Community College (WCC). Each week, students wrote reflectively to contrast their initial and current understanding of a specific physics topic, and described the "trigger" events that led them to change their thinking. Goals were to enhance conceptual understanding as well as the depth and quality of student reflection. A coding scheme was developed to evaluate student reflections. We present the scaffolded activity and coding scheme, as well as preliminary findings about changes in student reflection over time and correlations between amount of reflection and conceptual learning.
\end{abstract}

Keywords: reasoning, metacognition, laboratory, reflections.

PACS: $01.30 . \mathrm{Cc}$

\section{INTRODUCTION}

It has been shown that experts tend to actively monitor their own progress in learning new content and solving problems, while novices do not $[1,2]$. Additional research shows that the progression from novice to expert generally requires extensive practice $[3,4]$. The study described here is a part of an ongoing effort to explore how the transition from novice to expert-like thinking in physics can be expedited by actively promoting student metacognition.

In order to narrow the vast scope encompassed by metacognition, we have focused on reflective thinking, a "backward-looking" form of metacognition in which students revisit their initial ideas, describe specific changes in their understanding, and discuss the learning events that triggered those changes. We hypothesize that deliberate analysis of the learning process will solidify and deepen content understanding while also promoting the development of thinking habits more closely aligned with those of experts.

This work builds on an emerging body of research on student reflection in physics [5-9] and is inspired, in part, by the success of guided inquiry curricula in promoting conceptual understanding [10]. We are adding to this research by developing methods for identifying and categorizing instances of metacognition that occur as students reflect on their learning of specific concepts. We have implemented a weekly, scaffolded activity that guides students through the process of reflecting on their own learning in the laboratory component of an introductory calculusbased physics course. We have also designed the Metacognitive Elements Rubric (MER) to systematically evaluate the narrative reflections that students write as part of the activity. The impact of reflection was probed by examining correlations between MER scores and normalized gain scores on the Force Concept Inventory (FCI) [11].

\section{RESEARCH METHODS}

Research was conducted in the introductory calculus-based mechanics course at WWU. (Although the structured reflection assignment is used at WCC, a full data set has not yet been collected there.) This course consists of four one-hour lectures and a required three-hour lab. Labs are taught by undergraduate physics majors, with overall supervision and weekly TA preparation provided by physics faculty. During lab, students work in collaborative small groups through sequenced experiments and questions. The labs emphasize the development of conceptual understanding, with many activities adapted from Tutorials in Introductory Physics [12]. Students complete a set of 7 labs in kinematics and dynamics during the 10 -week quarter.

The sample population for this study was drawn from a single lecture section in which the FCI was administered. Students were included in the sample if they 1) took the FCI both pre- and post-course, and 2) completed all 7 required labs. While 65 students were enrolled in the course, only 42 took both the pre- and post-FCI, and only 17 of these 42 completed all 7 labs.

Weekly reflection assignment. The weekly reflection assignment occurs in the context of the lab. The lab sequence begins with the prelab, a set of written elicitation questions that students complete individually. After working through lab activities targeting the relevant physics content, students 
collaboratively review their initial responses to the prelab. Students annotate their prelab in order to indicate any changes in their answers or reasoning.

In lab homework, students write a 2-4 paragraph narrative reflection in which they compare their current and initial thinking. Carefully designed prompts guide students through the process of reflection. Students are asked to a) identify any aspects of their initial prelab reasoning they now regard as problematic; b) discuss what underlying difficulties may have led to the incorrect responses they identified, c) describe specific aspects of their current understanding that are newly formed or modified; d) discuss specific experiences in the lab that led to the new or modified understanding; and e) rate their comfort level with the concepts and identify any remaining questions. Students receive credit based simply on whether or not they address the above prompts (rather than on the perceived quality of their reflection). TAs provide written feedback on the reflections to encourage students to be explicit and specific in discussions of their physics understanding.

Data collection. Students' annotated prelabs and written reflections were scanned each week. The FCI was administered in lecture under exam conditions on the second day of class and again on the last day of class. Normalized gain scores were computed in the standard manner: $g=($ post - pre $) /(100 \%-$ pre $)$.

Assessing student reflection. The Metacognitive Elements Rubric (MER) was developed to systematically evaluate students' written reflections. Due to the inherent subjectivity in assessing the quality of reflection, we chose to focus simply on categorizing the type of statement made by the student. The rubric has 13 individual codes clustered into four groups:

Code Group A (Cognition):

1-Statement of initial prelab answer; 2-Statement of initial prelab reasoning; 3-Statement of current answer; 4-Statement of current reasoning

Code Group B (Reflection on initial ideas): 5-Statement that identifies an answer, explanation, or idea as incorrect or incomplete; 6-Statement that explains what is incorrect about the idea or describes how it is incomplete; 7-Statement that discusses what underlying difficulty led to the incorrect idea.

Code Group C (Reflection on current understanding): 8-Statement that identifies a concept, idea, skill, or reasoning element as newly learned or better understood; 9-Discussion or explanation of the new or improved knowledge; 10-Statement that illustrates or applies a newly learned concept or idea,.

Code Group D (Metacognition):

11-Any combination of statements that explicitly relates the student's initial and current ideas; 12-Statement that identifies a specific event or activity as having caused or provided impetus for a change in thinking; 13-Statement that identifies a specific concept that is still difficult to understand or apply.

A phrase, sentence, or group of sentences in a student's narrative reflection can receive a single code, multiple codes, or no code at all. Statements that would not receive a code include: "I learned a lot," "my answer was wrong," and "the lab helped me understand acceleration." The student reflection below, about the kinematics of a pendulum, identifies coded statements in italics and by code number.

"My original thinking was that as an object moves, the acceleration vector will follow the same path as the velocity vector (2). However in lab I found out that this is not the case (5). Instead the acceleration vector moved along the path of the change in velocity (8). This somewhat makes sense to me because in previous labs, I described the change in velocity or the derivative of velocity to be acceleration, it would only make sense that the change in velocity would describe the acceleration direction as well (9). On my prelab, I said 'The acceleration vectors should have the same magnitude, although the direction is changing' (1). At least some of that was correct in that the object's acceleration is constant, but the better way to phrase the direction of the acceleration (5) would have been to say that the change in the angular velocity displays the direction of the acceleration vector $(9,11)$..."

The MER is designed to operationalize reflective thinking and to be used to identify how much and what type of reflection students engage in. However, we also recognize its limitations: the MER allows measurement of the rate of occurrence of different elements of metacognitive reflection, but does not track the quality or accuracy of student reflection per se. For example, in some cases we find two statements that receive the same code but are of clearly different quality, while in others, a student's description of her initial understanding seems inconsistent with her written prelab explanation.

To establish reliability of the MER, two researchers completed multiple cycles of testing in which they independently coded several student reflections, compared results, resolved discrepancies through discussion, and then in some cases modified the rubric. After this process, a different pair of researchers used the revised MER to independently code a set of 20 previously uncoded student reflections. Comparison revealed complete agreement on which student statements should receive a code, and about $85 \%$ agreement on which of the 13 codes should be assigned to those statements. We note that a subsequent discussion to reconcile coding differences quickly resulted in full agreement. 
Data analysis. After establishing reliability, a single researcher coded the narrative reflections written by the 17 students in the sample for each lab 2-7, for a total of 102 coded narrative reflections. Lab 1 was excluded on the assumption that many students would require a full lab sequence to become oriented to the assignment. Various measures have been computed to summarize the resulting code data, including 1) the total number of coded statements made by each student (summed over all 6 of the narrative reflection assignments), 2) the total number of coded statements occurring on each of the 6 narrative reflections (summed over all 17 students), and 3) the fraction of the total number of coded statements represented by the number of instances of either an individual code (e.g., Code 12) or of any individual code within a particular code group (e.g., Code Group D).

\section{RESULTS}

The average normalized FCI gain of the 17 students in the sample was 0.73 , while the average gain for the group of all students who took the FCI was 0.66 . We speculate that the criterion of completing all 7 labs may have selected for more committed (and perhaps academically stronger) students. We explain the overall high gains by noting that the instructor had more than 10 years of experience with interactive engagement teaching methods.

The total number of codes produced by an individual student over all 6 written reflections ranged from 29 to 95 , with an average of 54 codes per student. Conversely, the aggregate number of codes produced by the group of 17 students ranged from a low of 125 in Lab 2 (Acceleration in One Dimension) to a high of 180 in Lab 5 (Newton's $2^{\text {nd }}$ law). Figure 1 shows the aggregate number of codes for each lab. We see that this number was below the average value for Labs 2-4 and above the average for Labs 5-7, suggesting that students gained proficiency with (or at least clarity about) the assignment as the course progressed.

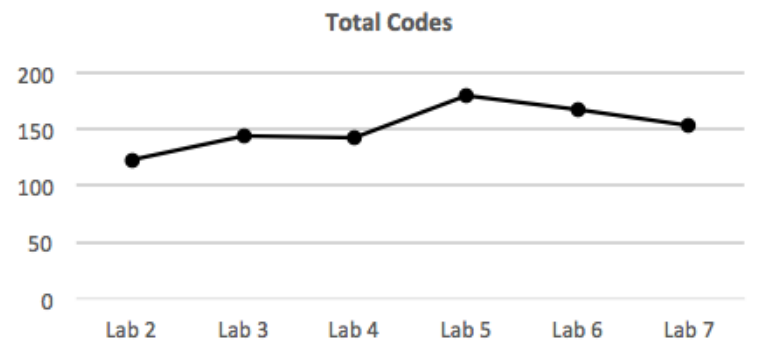

FIGURE 1. Number of MER codes by lab.

In addition to examining the total number of codes, we tracked the occurrence rates of individual codes. For example, on the narrative reflection for lab 2, code
5 represented $13 \%$ of all codes produced by the group of 17 students. This fraction varied from $8 \%$ to $14 \%$ over the 6 assignments. The range of variation was similar for other codes. These rates, which are nonzero and somewhat stable over time, suggest that students do, in fact, engage in the type of backwardlooking metacognitive reflection that the weekly assignment is designed to foster. We note that using occurrence rates, rather than total numbers of occurrences, controls for differences due to content. It may be that some lab topics are more conducive to reflection, leading to a higher total number of codes (e.g., lab 5 vs. lab 7).

In the scatter plots in Fig. 2, each point represents one student. In the panel on the left, the horizontal axis plots normalized FCI gain while the vertical axis shows the total number of MER codes produced by the student over all 6 reflections. Results suggest the lack of a clear relationship between these two variables, with a Pearson correlation coefficient of $\mathrm{R}=0.008$. Students who produced more coded statements were no more or less likely to achieve high FCI gains than students who produced fewer codes.

In addition to the total number of codes, we have examined correlations with the fraction of total codes represented by specific code groups. This was computed for a given student and a given code group as the ratio of the number of instances of any code from the code group to the overall number of coded statements. For example, a student with 70 coded statements in the 6 narrative reflections, 16 of which were from group A, would have a fraction of $24 \%$.

Correlation coefficients with FCI gains were computed for the proportion of each of the code groups. Groups B and D showed no correlation, group $\mathrm{A}$, a negative correlation, and $\mathrm{C}$, a positive correlation. The two panels on the right in Fig. 2 show the correlations for code groups A and C, respectively.

The negative correlation in the middle panel has Pearson $\mathrm{R}=0.47$ (two-tailed $\mathrm{p}=0.056$ ). This suggests that students who spend a greater fraction of their reflection on the elements related to initial answers or explanations, which represents what we consider a shallow level of reflection, tend to exhibit lower gains on the FCI. These students may be spending less time and effort on deeper modes of reflections likely to be associated with greater content learning (e.g. how their reasoning was flawed, what underlying confusion or difficulty led to specific mistakes, and what specific evidence led them to revise their thinking). However, we regard this claim as tentative due to a small sample of only 17 students. Removing the three outlying students reduces the correlation coefficient to $\mathrm{R}=0.17$. Despite the tentative nature of this result, we believe it suggests future directions for research. 

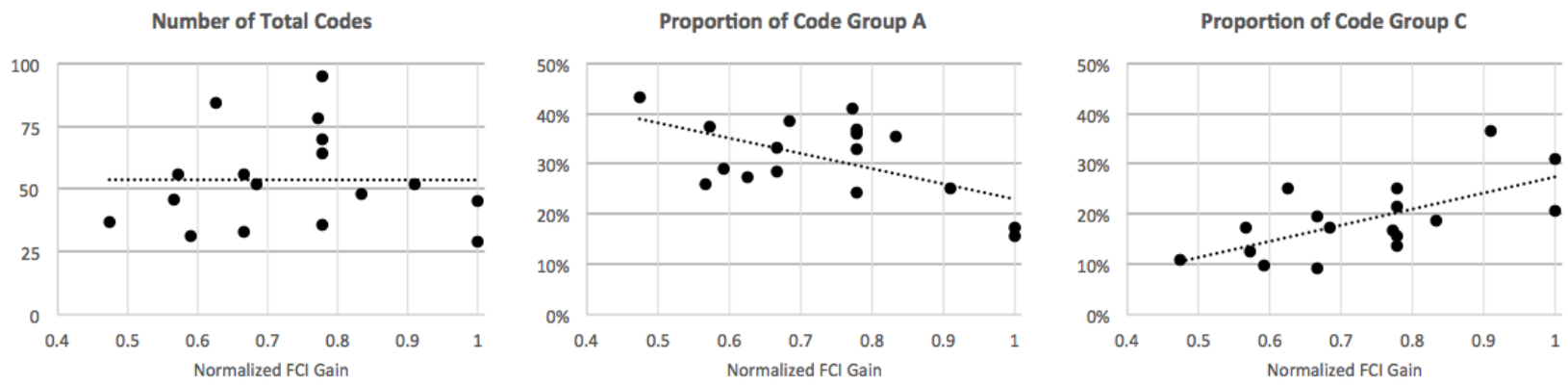

FIGURE 2. Correlations between normalized FCI gains and numbers or proportions of MER codes. Note truncated scale on horizontal axis.

The right panel of Fig. 2 represents a significant correlation $(\mathrm{R}=0.62$, two-tailed $\mathrm{p}=0.008)$, suggesting students who reflected more on their current understanding tended to achieve greater FCI gains. We note, however, that one of the group $\mathrm{C}$ codes embodies a metacognitive element not explicitly asked for in the assignment prompt (code 10, involving illustrating a concept). Narratives including this code may signal students with high intrinsic motivation to examine their learning, which may in turn affect FCI gain.

\section{DISCUSSION}

The results presented above do not provide strong evidence of a relationship between the amount of student reflection and increases in conceptual understanding. Several explanations are considered, with each providing directions for future research. First, it is possible that the narrative reflection assignment facilitates student learning in ways that the employed instruments (the FCI and the MER) are not sophisticated enough to capture. For example, while the MER seems effective in documenting the types of reflection students engage in, it is not designed to gauge the quality of that reflection. It may be that within the range of student statements that fall under a given code, only certain types are strongly linked to enhanced learning. Second, it may be that the assignment itself provides too heavy of a scaffold. Although students are able to complete the steps as prompted, their overall effort may remain, in essence, in the realm of "answer-making," rather than becoming an authentic experience of evaluating their own learning. We are currently testing a more openended reflection prompt to explore this possibility. Finally, as noted above, the FCI gains of the students included in this study are higher than what is typically measured in introductory physics. Indeed, with gains ranging from 0.49 to 1 , it is possible that a ceiling effect has suppressed the relationship between amount of reflection and FCI gains.

\section{CONCLUSIONS}

A scaffolded writing assignment has been developed in which students reflect on how they came to understand a specific physics topic or idea. A rubric for categorizing the reflective statements made by students in response to the assignment has been developed and tested. Preliminary results suggest that the assignment can lead students to reflect in specific desirable ways on their own learning. Further directions for research include a scaled-up study that we anticipate will provide the statistical power necessary to further explore the impact of student reflection on conceptual learning.

\section{ACKNOWLEDGEMENTS}

The authors gratefully acknowledge NSF support (DUE-1245993, DUE-1245999, DUE-1156974, and DUE-1245699).

\section{REFERENCES}

1. M. S. Donovan, J. D. Bransford, and J. W. Pellegrino, How People Learn: Bridging Research and Practice (The National Academies Press, 1999).

2. B. Y. White and J. R. Frederiksen, Cognition and Instruction 16, pp. 3-118 (1998).

3. K. A. Ericsson, M. J. Prietula, and E. T. Cokely, Harvard Bus. Rev. 85 (7-8), pp. 114-121 (2007).

4. A. H. Schoenfeld, Cognitive Science and Mathematics Education, pp. 185-215 (1987).

5. E. Yerushalmi, E. Cohen, A. Mason, and C. Singh, Phys. Rev. ST Phys. Educ. Res. 8, 020109-(2012).

6. E. Yerushalmi, E. Cohen, A. Mason, and C. Singh, Phys. Rev. ST Phys. Educ. Res.8, 020110-(2012).

7. D. B May \& E. Etkina, Am. J. Phys. 70, (2002).

9. R. Lippmann Kung and C Linder, Metacognition and Learning, Springer, pp. 41-56 (2007).

10. R. Hake, Am. J. Phys. 66, pp. 64-74 (1998).

11. D. Hestenes, M. Wells \& G. Swackhammer, The Physics Teacher 30 pp. 141-151, (1992).

12. L. C. McDermott and P. S. Schaffer, Tutorials in Introductory Physics, $2^{\text {nd }}$ ed., (Prentice Hall, 2002). 